\title{
Interactions between Circadian Neurons Control Temperature Synchronization of Drosophila Behavior
}

\author{
Ania Busza, ${ }^{1,2}$ Alejandro Murad, ${ }^{1}$ and Patrick Emery ${ }^{1}$ \\ ${ }^{1}$ Department of Neurobiology and Program in Neuroscience and ${ }^{2} \mathrm{MD} / \mathrm{PhD}$ Program, University of Massachusetts Medical School, Worcester, \\ Massachusetts 01605
}

\begin{abstract}
Most animals rely on circadian clocks to synchronize their physiology and behavior with the day/night cycle. Light and temperature are the major physical variables that can synchronize circadian rhythms. Although the effects of light on circadian behavior have been studied in detail in Drosophila, the neuronal mechanisms underlying temperature synchronization of circadian behavior have received less attention. Here, we show that temperature cycles synchronize and durably affect circadian behavior in Drosophila in the absence of light input. This synchronization depends on the well characterized and functionally coupled circadian neurons controlling the morning and evening activity under light/dark cycles: the $\mathrm{M}$ cells and $\mathrm{E}$ cells. However, circadian neurons distinct from the $\mathrm{M}$ and $\mathrm{E}$ cells are implicated in the control of rhythmic behavior specifically under temperature cycles. These additional neurons play a dual role: they promote evening activity and negatively regulate E cell function in the middle of the day. We also demonstrate that, although temperature synchronizes circadian behavior more slowly than light, this synchronization is considerably accelerated when the $\mathrm{M}$ cell oscillator is absent or genetically altered. Thus, whereas the E cells show great responsiveness to temperature input, the M cells and their robust self-sustained pacemaker act as a resistance to behavioral synchronization by temperature cycles. In conclusion, the behavioral responses to temperature input are determined by both the individual properties of specific groups of circadian neurons and their organization in a neural network.
\end{abstract}

Key words: circadian rhythms; behavior; temperature synchronization; neural network; circadian neurons; morning and evening oscillators

\section{Introduction}

Like most organisms, Drosophila melanogaster uses a circadian clock to synchronize its physiology and behavior with the day/night cycle. Many of the molecular and cellular components of this internal pacemaker are identified. The products of the period ( per), timeless (tim), clock (clk), and cycle genes form a transcriptional feedback loop; a set of kinases and phosphatases adjusts the period of this oscillator to $24 \mathrm{~h}$ (Hardin, 2005). Intracellular signaling through the photoreceptor CRYPTOCHROME (CRY) and synaptic input from visual organs synchronize the circadian clock to the light cycle (Helfrich-Förster et al., 2001). Drosophila exhibit a bimodal locomotor activity pattern under a light/dark (LD) cycle, with morning (M) and evening (E) surges of activity. Specific circadian neurons contribute to these peaks of activity (Grima et al., 2004; Stoleru et

\footnotetext{
Received 0ct. 2, 2006; revised Aug. 21, 2007; accepted Aug. 21, 2007.

This work was supported by National Institutes of Health (NIH) Cellular and Molecular Neurobiology Training Grant T32 NS07366 and NIH-National Institute of Neurological Disorders and Stroke Kirschstein National Research Service Award 5 F30 NS054421-01 (A.B.) and NIH R01 Grant 5R01GM066777 (P.E.). We thank Dan Stoleru and Michael Rosbash for the pdf-GAL80 flies and anti-PER and anti-PDF antibody; Francois Rouyer for per ${ }^{0}$ w; + ; UAS-per flies and the FAAS software; and Joel Levine for the Matlab analysis toolbox and Ralf Häfner for help streamlining the Matlab analysis. We thank Dave Weaver and Michael Rosbash for critical reading of this manuscript. We are also grateful to members of the Emery, Weaver, and Reppert laboratories for helpful discussions. We thank Diane Szydlik for technical help.

Correspondence should be addressed to Patrick Emery, Department of Neurobiology and Program in Neuroscience, University of Massachusetts Medical School, 364 Plantation Street, Worcester, MA 01605. E-mail: patrick.emery@umassmed.edu.

D0I:10.1523/JNEUROSCI.2479-07.2007

Copyright $\odot 2007$ Society for Neuroscience $\quad$ 0270-6474/07/2710722-12\$15.00/0
}

al., 2004, 2005; Yoshii et al., 2004; Rieger et al., 2006). The ventral lateral neurons (LNvs) control the morning peak and are consequently also referred to as "M cells." The dorsal lateral neurons (LNds), the pigment dispersing factor (PDF)-negative LNv, and possibly some dorsal neurons 1 (DN1s) are responsible for the evening peak and are thus called " $\mathrm{E}$ cells” (Grima et al., 2004; Stoleru et al., 2004; Rieger et al., 2006). Other circadian cell groups include the lateral posterior neurons (LPNs) and other dorsal neurons (DN2s and DN3s), about which much less is known (Kaneko and Hall, 2000; Shafer et al., 2006).

Intriguingly, environmental inputs affect the relative contribution of different brain neurons to circadian activity, and this regulation may aid Drosophila to adapt to seasonal changes in day length (Miyasako et al., 2007; Murad et al., 2007; Stoleru et al., 2007). Under constant darkness (DD), the LNvs are necessary and sufficient for behavioral rhythms (Renn et al., 1999; Grima et al., 2004) and dictate their period (Stoleru et al., 2005). However, genetic studies revealed that, under constant light (LL), the E cells and a subset of DN1s can also function as pacemaker neurons (Murad et al., 2007; Stoleru et al., 2007). Moreover, the respective contribution of the $\mathrm{M}$ and $\mathrm{E}$ cells to the control of circadian behavior depends on the length of the photoperiod (Stoleru et al., 2007). In addition, separate cell groups may be differentially sensitive to temperature or light cycles when these cues are simultaneously present (Miyasako et al., 2007).

Most of the work on how Drosophila synchronizes its clock to environmental cycles has centered on light input pathways. How- 
ever, temperature fluctuations can also reset circadian clocks. Temperature can dominate light input in Neurospora (Liu et al., 1998). In mammals, temperature cycles mimicking body temperature fluctuations help keep peripheral clocks synchronized (Brown et al., 2002). In Drosophila, temperature cycles synchronize eclosion rhythms (Pittendrigh, 1954), locomotor activity rhythms (Wheeler et al., 1993; Yoshii et al., 2002, 2005; Glaser and Stanewsky, 2005), and molecular oscillations in peripheral tissues (Glaser and Stanewsky, 2005) and brain neurons (Yoshii et al., 2005). To understand further how temperature synchronizes Drosophila behavior, we studied the contribution of different circadian neurons and the intercellular network connecting them to thermal entrainment.

\section{Materials and Methods}

Drosophila strains and transgenics. Flies with targeted neuronal ablation of the $\mathrm{M}$ cells or both the $\mathrm{M}$ and $\mathrm{E}$ cells were obtained as described previously (Renn et al., 1999; Stoleru et al., 2004) from the following stable stocks: $y$ w; UAS-hid/CyO; + flies, $y w$; pdf-GAL4; +, and $y w$; +; cry-GAL4-13/TM6b. The $p d f^{01}$ mutant flies were described previously (Renn et al., 1999). For neuronal ablation in per mutants, UAS-hid was introduced into $\mathrm{per}^{O}, \mathrm{per}^{S}$, and $\mathrm{per}^{L}$ backgrounds, and these lines were then crossed to $y w$; pdf-GAL4; + or $y w ;+; c r y-G A L 4-13 / T M 6 b$ flies. $p^{0}{ }^{0}$ flies were rescued by expressing per with the $p d f-G A L 4$ driver as described previously (Grima et al., 2004), as well as with cry-GAL4-13 and a $c r y-G A L-13 / p d f-G A L 80$ combination (Stoleru et al., 2004). To make $y$ w; pdf-GAL4/+;UAS-per/+ flies, we crossed $y w$; pdf-GAL4 virgin females with $\operatorname{per}^{0} w$; +; UAS-per males (Grima et al., 2004). Clk ${ }^{J r k}$ heterozygote flies were made by crossing $y w$ females with $+;+; \mathrm{Clk}^{\text {rk }} / T M 2$ males (Allada et al., 1998).

Behavioral assays and analysis. To record daily locomotor activity, individual male flies (1-6 d old) were placed into Drosophila activity monitors (Trikinetics, Waltham MA). All experiments were done in Percival I-36LL incubators (Percival Scientific, Perry IA). The thermophase/ cryophase (TC) cycles were performed at a temperature of $29^{\circ} \mathrm{C}$ for the thermophase and $20^{\circ} \mathrm{C}$ for the cryophase. Temperature during runs was monitored with a Fluke SII 53 digital thermometer. Shifting temperature from $20^{\circ} \mathrm{C}$ to $29^{\circ} \mathrm{C}$ took $\sim 30 \mathrm{~min}$ in our incubators. Once the system had reached the correct temperature, it remained stable within $\pm 0.4^{\circ} \mathrm{C}$. A light intensity of $\sim 150-200$ lux was used for LD cycles and for one of the LL experiments. For the other (LL) experiment, some light bulbs were turned off in the incubator to obtain a light intensity of $\sim 75$ lux. For each experiment, details on the number of days in LD, TC, and constant conditions (CC) $\left(\right.$ dark, $\left.20^{\circ} \mathrm{C}\right)$ are explained in the text or in the figure legends.

Behavior data collected with the Drosophila Activity Monitoring program (Trikinetics) was analyzed with FaasX (courtesy of F. Rouyer, Centre National de la Recherche Scientifique, Gif-sur-Yvette, France) or a signal processing toolbox for Matlab (MathWorks, Natick, MA) (courtesy of J. Levine, University of Toronto, Mississauga, Ontario, Canada). All actograms are group averages plotted using Matlab "dam_panels" function (Levine et al., 2002). Histograms are group averages plotted using Matlab "dam_panels" function (see Fig. 7) (supplemental Fig. S5, available at www.jneurosci.org as supplemental material) or are group average eductions using FaasX (see Figs. 4, 6).

To determine the phase of the daily evening peak before, after, or during temperature cycles (see Figs. $1 B, 8 A, B$ ) (supplemental Fig. S1, available at www.jneurosci.org as supplemental material), we analyzed all flies that had survived the entire run. Phase was calculated for each individual fly for each day in Matlab with the "peakphaseplot" function (using an $8 \mathrm{~h}$ Butterworth filter and manual removal of non-evening peaks), and the group mean and SE was calculated and plotted in Excel (Microsoft, Seattle, WA).

For experiments measuring the phase shift of TC-entrained circadian behavior after release into CC (Figs. 1C, 2, 8C) (supplemental Fig. S3, available at www.jneurosci.org as supplemental material), we calculated the phase of the evening peak on the second day after release in CC for each experimental group. To calculate relative phase shift of the TCexposed group of flies, mean phase of the no-TC control group was subtracted from mean phase of the TC-exposed group, and the combined SE of the two groups of flies was calculated. Arrhythmic flies were excluded from all phase-shift analysis except in Figure $8 C$, for which all flies with a clear evening peak on the second day were used.

Immunohistochemistry. Brain immunostainings for PER and PDF were performed as described by Murad et al. (2007).

\section{Results}

Temperature is a Zeitgeber for circadian locomotor rhythms To study the neuronal mechanisms underlying synchronization of Drosophila behavior by temperature cycles, we decided to perform the majority of our experiments in DD. These conditions allow us to study specifically temperature synchronization without the confounding effects of light input and also to monitor the impact of temperature cycles on the circadian pacemaker underlying rhythmic behavior. This cannot be done under LL, which was used in past studies (Glaser and Stanewsky, 2005; Yoshii et al., 2005), because circadian rhythms immediately degenerate under LL conditions after return to constant temperature.

Temperature cycles of as little as $3^{\circ} \mathrm{C}$ have been shown to synchronize locomotor activity in DD (Wheeler et al., 1993). To verify that this is attributable to a genuine effect on the circadian clock as opposed to a temporary "masking" effect of temperature variations on behavior, we looked for changes in circadian phase that persist after temperature entrainment. Using $12 \mathrm{~h} / 12 \mathrm{~h} 29^{\circ} \mathrm{C} /$ $20^{\circ} \mathrm{C}$ thermophase/cryophase temperature cycles in $\mathrm{DD}$, we phase-advanced or phase-delayed wild-type flies (Fig. $1 A$ ) that had been previously synchronized to an LD cycle. After several days of TC, we observed a robust evening peak of activity that anticipated the temperature transition, suggesting that the circadian clock underlying the evening activity had been re-entrained by the temperature cycle. Anticipation of the morning temperature transition was visible in some of our experiments (for example, Fig. $1 A$, right) but was usually of much smaller amplitude than that observed in an LD cycle and sometimes not detectable. This might be because morning activity is suppressed at lower temperatures, as shown previously in LD studies at different constant temperatures (Miyasako et al., 2007). Therefore, to begin our analysis of the phase-shifting effects of TC cycles on circadian behavior, we measured each fly's daily evening peak and compared it with the evening peaks of control flies left in constant conditions $\left(20^{\circ} \mathrm{C} \mathrm{DD}\right)$ (Fig. $\left.1 \mathrm{~B}\right)$. On the last day of LD, the fly's evening activity peaked at approximately Zeitgeber time 12 (ZT12) (in which ZT0 refers to the lights-on time during LD). The evening activity peak then drifted to approximately ZT11 by the fourth day of DD (day 6). After $5 \mathrm{~d}$ of TC, flies exposed to a $9 \mathrm{~h}$ advanced TC cycle had evening peaks $8 \mathrm{~h}$ earlier than the control flies, showing that their behavior had been resynchronized. Conversely, flies exposed to a $6 \mathrm{~h}$ delay TC cycle had evening peaks $6 \mathrm{~h}$ later than controls. All fly groups showed a daily advance when released into constant $20^{\circ} \mathrm{C}$ DD because our $y w$ wild-type flies have a period slightly shorter than $24 \mathrm{~h}$ in constant conditions. Importantly, however, the phase advance and delay of the TCexposed groups is maintained after releasing the flies into constant conditions. This demonstrates that the circadian clock, and not just behavior, has been synchronized by the temperature cycles. Temperature is thus a Zeitgeber (time-giver) for adult circadian behavior, i.e., it is an input that can durably affect the phase of circadian behavior, even after return to constant conditions. It also provides evidence that temperature fluctuations can affect the clock in the absence of any light input. Interestingly, synchronization under a TC cycle is much slower than under an LD cycle. 
With an $8 \mathrm{~h}$ advanced TC, it takes over $6 \mathrm{~d}$ to reach a completely stable behavior phase, whereas it takes $5 \mathrm{~d}$ with an 8 h delay (supplemental Fig. S1, available at www. jneurosci.org as supplemental material). Light synchronization is significantly faster and takes at most $2 \mathrm{~d}$ (supplemental Fig. S2, available at www.jneurosci.org as supplemental material), at least under our experimental conditions (200 lux during the day, total darkness during the night) and those of previous studies (Emery et al., 2000; Helfrich-Förster et al., 2001). An additional difference between light and temperature entrainment is the final phase of the evening peak of activity if the lights-on and the temperature-up transitions are used as time references. We found that, during a TC cycle in constant darkness, the evening peak is a few hours earlier than in an LD cycle. This is consistent with the fact that, in nature, temperature variations usually lag behind the light cycle (Boothroyd et al., 2007).

We observed slow displacement of the evening peak between day 1 and day 6 (transients) (supplemental Fig. S1 E, available at www.jneurosci.org as supplemental material). Wondering whether the phase of this peak reflects the state of synchronization of the underlying circadian pacemaker, we entrained flies for $1,2,3$, or $4 \mathrm{~d}$ to an $8 \mathrm{~h}$ advanced TC cycle and then released them in constant temperature to determine the phase of their free-running behavior (Fig. 1C). We also observed a progressive phase shift with increasing numbers of temperature cycles, similar to that observed with the evening peak under TC cycles (Fig. 1B) (supplemental Fig. S1E, available at www.jneurosci.org as supplemental material). Thus, the evening peak is a close reflection of the state of the underlying oscillator under TC cycles.

One more feature of the results shown on Figure 1 should be noted. On return to constant temperature, flies that have been advanced or delayed by temperature cycles shift their behavioral phase by $2-3 \mathrm{~h}$ toward the subjective morning. We presume that this phase change is attributable to the temperature-dependent per splicing that advances behavioral phase at $20^{\circ} \mathrm{C}$ and delays it at $29^{\circ} \mathrm{C}$ (it would thus do so during temperature entrainment) (Majercak et al., 1999). In addition, our $y w$ flies show short period rhythms. This probably explains why, on release to constant temperature, the peak of activity in $y w$ flies is shifted toward the early part of the day (see Figs. 1, 3, 6).

Depending on the relative time an animal is exposed to an environmental input, its circadian clock may advance, delay, or not respond to the stimulus (Bruce, 1960). For example, flies respond to short light pulses by delaying their clock after an early night pulse and by advancing it in response to a late night pulse. A light pulse during the subjective day does not phase shift the clock (Pittendrigh, 1967). To determine whether the Drosophila clock has a different response to a temperature cycle depending on when it experiences the warm temperature, wild-type flies en-
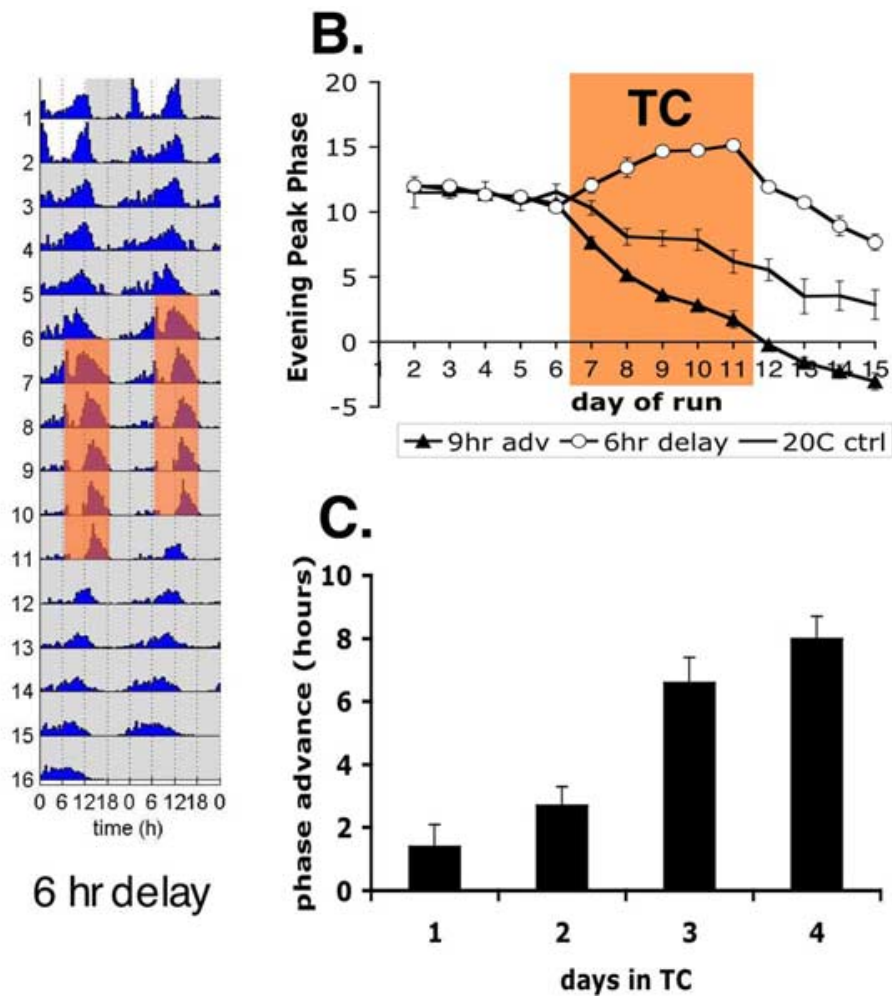

days in TC

Figure 1. Temperature is a Zeitgeber for Drosophila circadian behavior. $A$, Actograms showing the average locomotor behavior (maintained in CC after day 2) were used as controls $\left(20^{\circ} \mathrm{C} \mathrm{ctrl} ; n=11\right)$. The difference in post-TC phase was ( $y$-axis) represents the magnitude of the phase shift induced by the TC on the endogenous circadian oscillator (number of rhythmic flies ranged from 10 to 13 per fly group; rhythmicity ranged from 73 to 93\%).

trained at constant $20^{\circ} \mathrm{C}$ were exposed to $29^{\circ} \mathrm{C}$ for $12 \mathrm{~h}$ at different times of subjective night and day during constant darkness. The resulting phase shifts (relative to control flies continuously maintained at $20^{\circ} \mathrm{C}$ ) were graphed as a phase response curve (PRC) (Fig. 2A). Maximum phase shifts were elicited when the $29^{\circ} \mathrm{C}$ exposure began in late subjective day (delay of $3 \mathrm{~h}$ at ZT9 and ZT11) and in the mid/late subjective night (advance of 2-2.5 $\mathrm{h}$ at ZT17 and ZT19). Strikingly, $12 \mathrm{~h}, 29^{\circ} \mathrm{C}$ exposure beginning at ZT13 versus ZT15 elicits very different phase shifts ( $2 \mathrm{~h}$ delay and $1 \mathrm{~h}$ advance, respectively), despite $10 \mathrm{~h}$ of thermophase overlap. The $29^{\circ} \mathrm{C}$ exposure starting early in the subjective day (e.g., ZT1) elicited almost no phase shift, presumably because it coincided with the time of day when the animal expects its environment to become warmer. A $6 \mathrm{~h}$ pulse PRC was also generated and showed a similar shape with greater responses from pulses initiated at ZT11 and ZT19 (Fig. 2B), although the amplitude of the delay responses was smaller than with a $12 \mathrm{~h} 29^{\circ} \mathrm{C}$ pulse. Previous studies on Drosophila pseudoobscura eclosion rhythms show a similar phase-shifting curve in response to $12 \mathrm{~h}$ at $28^{\circ} \mathrm{C}$, with a reduced phase-delay during the subjective day (Zimmerman et al., 1968). That exposure to warm temperature can elicit responses with directionality and amplitude dependent on the state of the circa- 

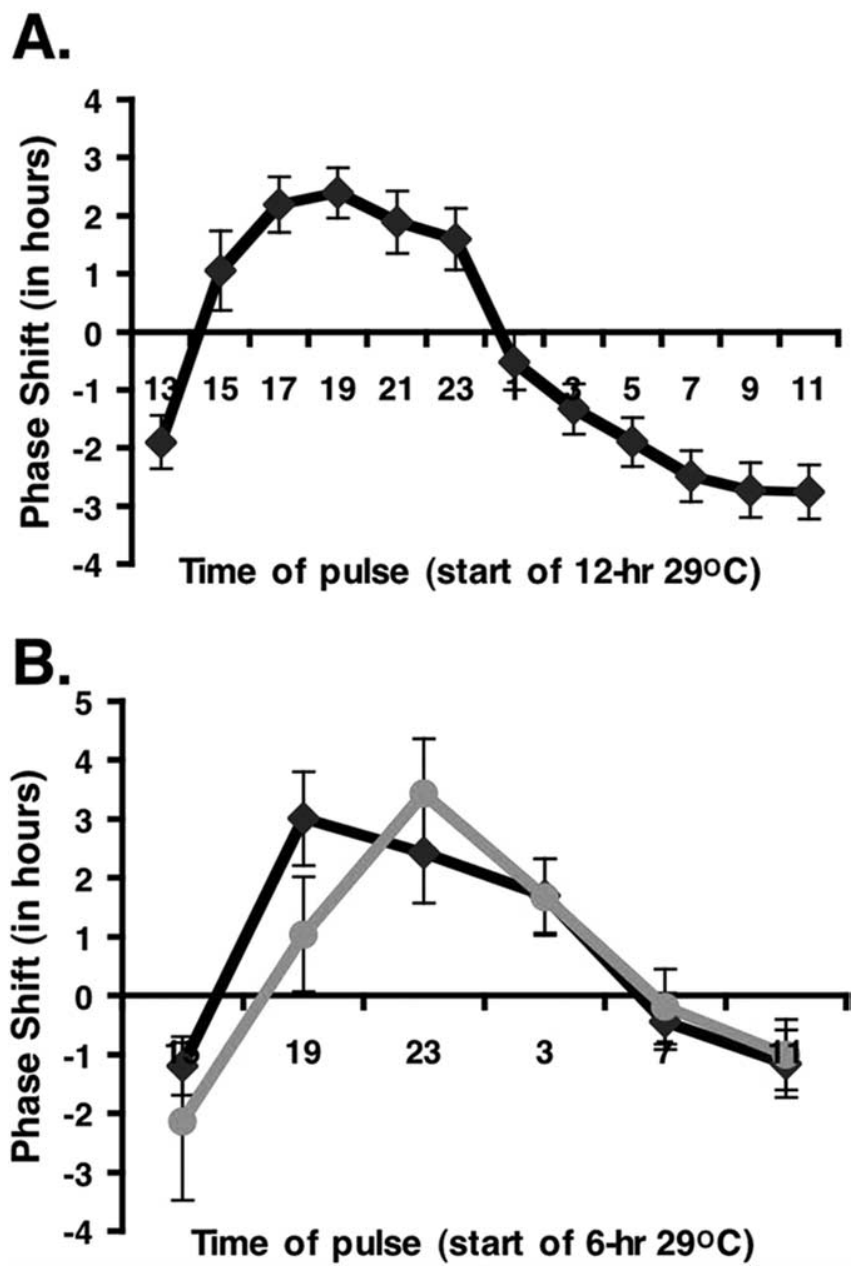

Figure 2. Phase response curve to 12 and $6 \mathrm{~h} 29^{\circ} \mathrm{C}$ warm pulses. $y w$ and $c r y^{b}$ flies were synchronized to an $L D$ cycle at $20^{\circ} \mathrm{C}$ and then exposed to $29^{\circ} \mathrm{C}$ for 6 or $12 \mathrm{~h}$ at different times of the night and the first subjective day. They were then kept in $\mathrm{DD}$ at $20^{\circ} \mathrm{C}$ to determine the phase of their locomotor behavior. $\boldsymbol{A}, y$ w flies (16-24 flies per time point) exposed to $12 \mathrm{~h}$ warm pulses. $\boldsymbol{B}, y$ w flies (black line; $9-15$ flies per time point) and $\boldsymbol{c r y}^{b}$ flies (gray line; $12-16$ flies per time point) exposed to $6 \mathrm{~h}$ warm pulses. $x$-Axis, Start time of the exposure to $29^{\circ} \mathrm{C}$, in circadian time. $y$-Axis, Phase shift (in hours) of the evening peak relative to control flies not exposed to $29^{\circ} \mathrm{C}$. Error bars indicate \pm SEM.

dian clock reinforces the notion that temperature is a proper Zeitgeber for circadian behavior in Drosophila.

It has been shown recently that CRY binds to the PER/TIM dimer to mediate specific behavioral responses to temperature, such as the phase-shifting effects of a $1 \mathrm{~h}$ high-temperature heat pulse $\left(37^{\circ} \mathrm{C}\right)$ (Edery et al., 1994; Kaushik et al., 2007). Under $29^{\circ} \mathrm{C} / 20^{\circ} \mathrm{C}$ TC cycles, we did not observe any obvious entrainment defects in $c r y^{b}$ mutant flies, although these flies have no functional CRY and do not respond to $37^{\circ} \mathrm{C}$ heat pulses (Stanewsky et al., 1998; Kaushik et al., 2007) (data not shown). We therefore decided to further challenge $c r y^{b}$ flies by exposing them to only a single 6 -h-long $29^{\circ} \mathrm{C}$ pulse. Both advance and delay responses to these single pulses were preserved in $c r y^{b} \mathrm{mu}-$ tant flies (Fig. $2 B$ ). We noticed a reduction in the advance response at ZT19, but, at ZT23, $c r y^{b}$ flies strongly phase advanced their clock. This result and previous molecular studies (Stanewsky et al., 1998; Glaser and Stanewsky, 2005) show that CRY is not essential for temperature entrainment of circadian rhythms at moderate temperature $\left(18-29^{\circ} \mathrm{C}\right)$ range. This suggests that, besides CRY, the PER/TIM dimer, proposed to be thermosensitive (Kaushik et al., 2007), can bind other molecules in a temperature-dependent manner to synchronize circadian rhythms with moderate temperature cycles. Higher temperature might either promote these putative interactions (as shown for CRY) or, on the contrary, destabilize complexes formed at low temperature.

The PDF-positive M cells are necessary for persistence of temperature-synchronized circadian behavior

Under an $\mathrm{LD}$ cycle, PDF-positive $\left(\mathrm{PDF}^{+}\right) \mathrm{LNv}$ are primarily responsible for the anticipatory behavior of flies at dawn and are thus called M cells, whereas a set of more dorsally located E cells are primarily responsible for the evening activity (Grima et al., 2004; Stoleru et al., 2004). In addition, the M cells maintain circadian rhythms in constant darkness (Renn et al., 1999; Grima et al., 2004). We used genetic techniques to ablate specific subsets of circadian neurons or rescue their clock function in an arrhythmic genetic background to determine the respective function of these groups of cells under dark TC cycles. To study the role of the $\mathrm{PDF}^{+}$cells, or M cells, we first ablated the LNvs by driving the proapoptotic gene hid (head involution defective) in these neurons with the $p d f$-GAL4 driver (Fig. 3B) (Renn et al., 1999). A brief surge of activity at the beginning of the thermophase was observed. Because it was present in all genotypes, even those without a functional clock, it is a noncircadian response to the temperature transition. No anticipation of the morning temperature change could be detected, but this absence of anticipation is not definitive proof that the $\mathrm{M}$ cells play a role in morning anticipatory activity under TC conditions because, even in wild-type flies, this activity cannot be reliably detected. In contrast, the surge of activity in late thermophase in the flies lacking $M$ cells strongly anticipates the temperature transition and is thus probably a circadian evening peak. This was also observed in a previous study using temperature cycles in constant light conditions (Yoshii et al., 2005). We will discuss the nature and the control of the evening peak in detail below. Importantly, there was no persistence of circadian behavioral rhythms on return to constant temperature (Fig. $3 B$ ). Thus, the $\mathrm{M}$ cells are critical for long-term behavioral rhythmicity after temperature synchronization. Because $p d f^{01}$ flies have the same phenotype as flies without $\mathrm{M}$ cells (Fig. 3C), the PDF neuropeptide is required for the function of the $\mathrm{M}$ cells under TC cycles.

\section{The $\mathrm{PDF}^{+} \mathrm{M}$ cells are sufficient for long-term} synchronization of circadian behavior after exposure to temperature cycles

To determine whether the $\mathrm{M}$ cells can independently maintain TC-entrained circadian behavioral rhythms, we rescued PER expression only in these cells in per $^{0}$ flies (Grima et al., 2004) (Fig. $3 E$ ). The midday peak seen in these mosaic flies is also seen in per $^{\circ}$ flies (Yoshii et al., 2002) (Fig. 3D). It is thus not generated by the rescued $\mathrm{M}$ cells but is actually caused by the improper activity of the E cells in the middle of the day because this peak is absent in flies without E cells (see below and Fig. 7). M-cell-rescued per $^{0}$ flies were able to remain rhythmic under constant dark after TC (Fig. 3E), whereas the per ${ }^{O}$ control flies could not (Fig. 3D). Notably, the activity was clearly resynchronized by the temperature entrainment. Therefore, a functional clock in the M cells is sufficient to maintain circadian rhythms after exposure to TC and for the phase-shifting effects of temperature cycles. These cells can thus receive information about temperature, but whether this information is obtained through a cell-autonomous thermoreceptor or synaptic input from peripheral sensors, or both, re- 

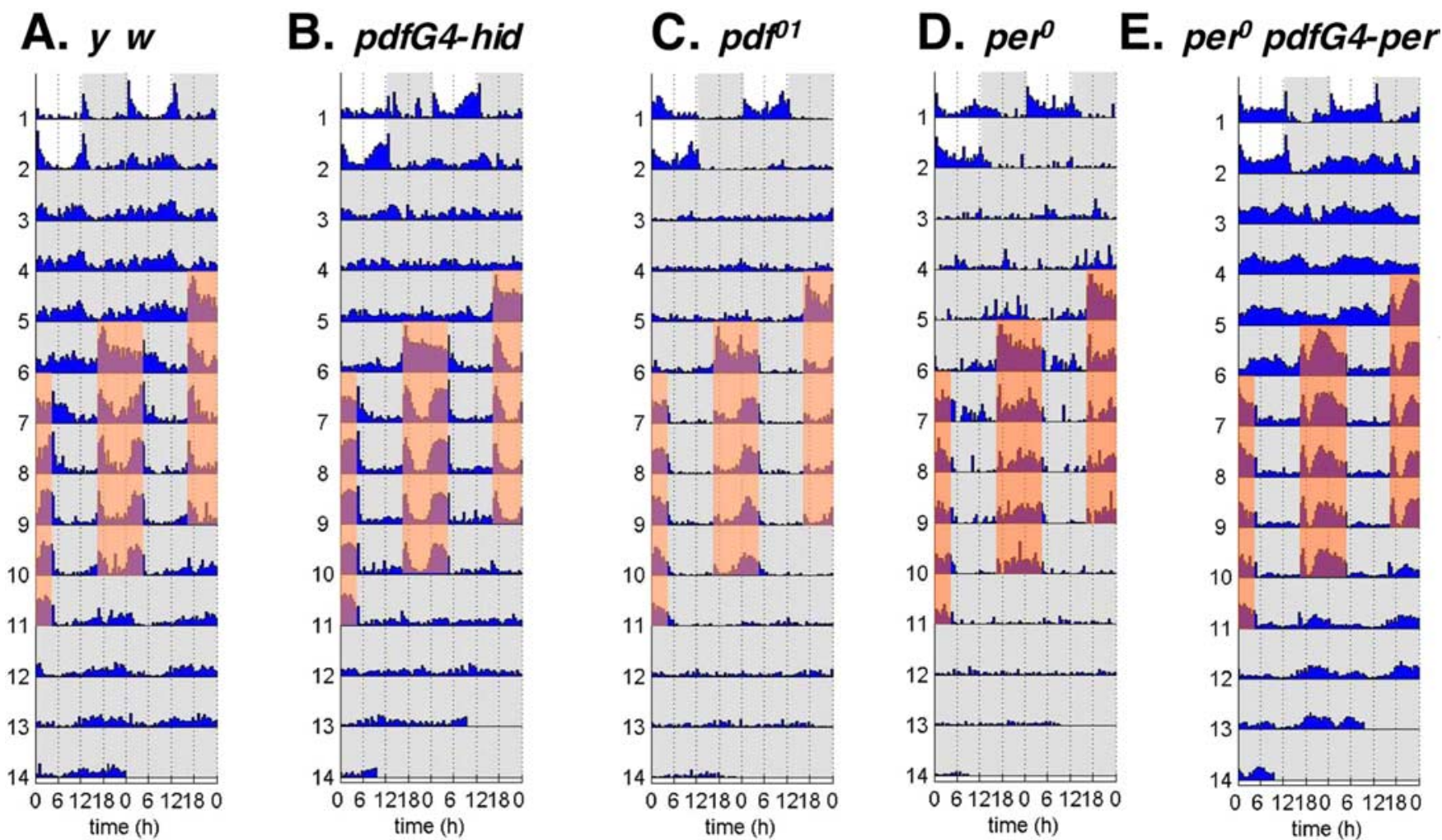

Figure 3. The PDF ${ }^{+} \mathrm{M}$ cells are necessary and sufficient for long-term synchronization of circadian behavior after exposure to temperature cycles. Flies with or without functional PDF ${ }^{+}$cells were exposed to $2 \mathrm{~d}$ of $20^{\circ} \mathrm{CLD}, 4 \mathrm{~d}$ of $\mathrm{CC}, 5 \mathrm{~d}$ of $29^{\circ} \mathrm{C} / 20^{\circ} \mathrm{C} \mathrm{TC}(8 \mathrm{~h}$ advance), and then $3 \mathrm{~d}$ of CC. $A$, Wild-type controls ( $y$ w; $+;+; n=12)$. B, pdfG4-hid, Flies without M-cells ( $y$ w; pdf-GAL4/UAS-hid; $+; n=15)$. C, Flies missing the neuropeptide PDF ( $\left.p d{ }^{\rho 1} ; n=30\right) . \boldsymbol{D}$, per $^{0}$, Flies with a null mutation in the per gene ( per $\left.^{0} ; n=6\right)$. $\boldsymbol{E}$, per ${ }^{0}$ pdfG4-hid, per flies with PER expression rescued only in the M cells ( per $^{0}$ w; pdf-GAL4/+;UAS-per/+; $n=16$ ). Note the persistence of circadian rhythms after TC in flies with the M cells being the only functional circadian neurons (E). Circadian rhythms are not maintained when these cells are either absent $(\boldsymbol{B})$ or do not produce $\operatorname{PDF}(\boldsymbol{C})$.

mains to be determined. We noted that the persistent activity after synchronization to a TC cycle was concentrated in the subjective morning, as observed after an LD cycle (Grima et al., 2004). This suggests that, similarly to what has been observed during or after an $\mathrm{LD}$ cycle, the $\mathrm{M}$ cells are mostly generating morning activity after exposure to TC cycles. Thus, the difficulty of detecting the anticipatory morning behavior under TC may be attributable to an inhibitory effect of the colder temperature (negative masking) (Miyasako et al., 2007) or possibly to the onset of this peak being slightly later in TC than in LD and thus positively masked by the surge of activity at the beginning of the thermophase.

The evening activity is controlled by the circadian clock and the $\mathrm{E}$ cells under temperature cycles

As mentioned above, an evening peak of activity is present in flies without $\mathrm{M}$ cells and in flies missing PDF under TC. A similar evening peak is seen in flies of the same genotypes exposed to an $\mathrm{LD}$ cycle, and it has been shown that, in DD, this peak persists for $\sim 2 \mathrm{~d}$ (Renn et al., 1999). For unknown reasons, we had difficulties detecting this short-term persistence of the evening peak after both $\mathrm{LD}$ and TC cycles when PDF or the M cells were missing. In some experiments, however, evening peak persistence after TC was clearly visible (supplemental Fig. S3, available at www.jneurosci.org as supplemental material), which suggests that the circadian clock controls this peak. To confirm that this peak is indeed regulated by components of the circadian system, we ablated the $\mathrm{M}$ cells in flies carrying arrhythmic or period-altering mutations in the circadian gene period: per $^{O}, \mathrm{per}^{S}$, and $\mathrm{per}^{\mathrm{I}}$
(Konopka and Benzer, 1971). To easily visualize the evening peak in the long period mutant, we used a temperature cycle with a longer day and a shorter night $\left(18 \mathrm{~h} 29^{\circ} \mathrm{C}, 6 \mathrm{~h} 20^{\circ} \mathrm{C}\right)$. The longer thermophase prevents the peak of activity of per $^{L}$ flies from occurring in the cryophase and being suppressed by cold phase negative masking, as it would in a standard $12 \mathrm{~h} / 12 \mathrm{~h} \mathrm{TC}$. Indeed, a peak is present in all three genotypes during the 18-h-long thermophase (Fig. 4). Its location relative to the temperature cycle is earlier in $\mathrm{per}^{S}$ than in wild-type flies but later in $\mathrm{per}^{L}$, demonstrating that the evening peak is gated by a per-dependent time-keeping mechanism. A peak of activity is also observed in $\operatorname{per}^{0}$ under this particular TC cycle, as well as under $12 \mathrm{~h} / 12 \mathrm{~h}$ TC (Fig. 3D) as described previously (Yoshii et al., 2002). The phase of this peak is abnormal; it is much earlier than the peak observed in any other per allele, even $\mathrm{per}^{S}$. It is eliminated when the $\mathrm{E}$ cells are ablated (see Fig. 7). Thus, the per-dependent circadian molecular machinery plays an important role in properly gating the activity of neurons controlling evening locomotor activity under TC cycles.

We then used a combination of cry-GAL4 and UAS-hid transgenes to create flies with both the $\mathrm{M}$ and $\mathrm{E}$ cells ablated (Stoleru et al., 2004). Immunocytochemical staining for PER was performed after TC entrainment to determine which circadian neurons were ablated (Fig. 5A). As described previously (Stoleru et al., 2004), the DN1s, DN2s, and DN3s are still present in these flies. However, the large number of DN1 and DN3 cells do not allow us to rule out that a subset of dorsal neurons is ablated. Based on Stoleru et al. (2004), it is likely that at least two DN1s are ablated. The LPNs were not ablated. As expected, the LNvs were missing. 
A. per ${ }^{+}$ pdfG4-hid
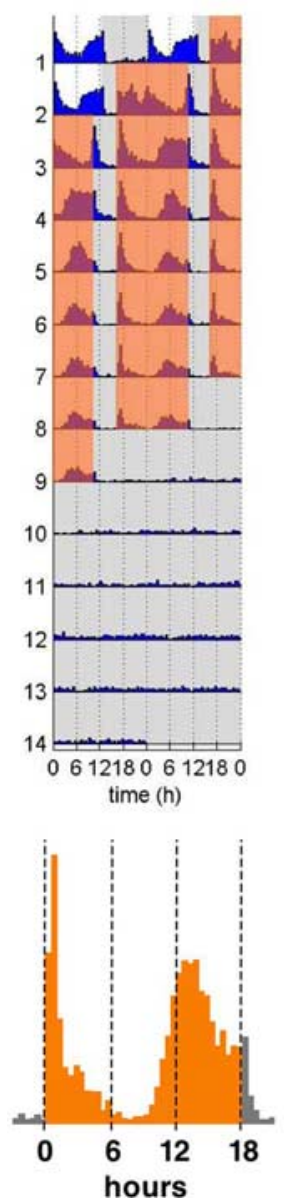

B. $p e r^{s}$ pdfG4-hid
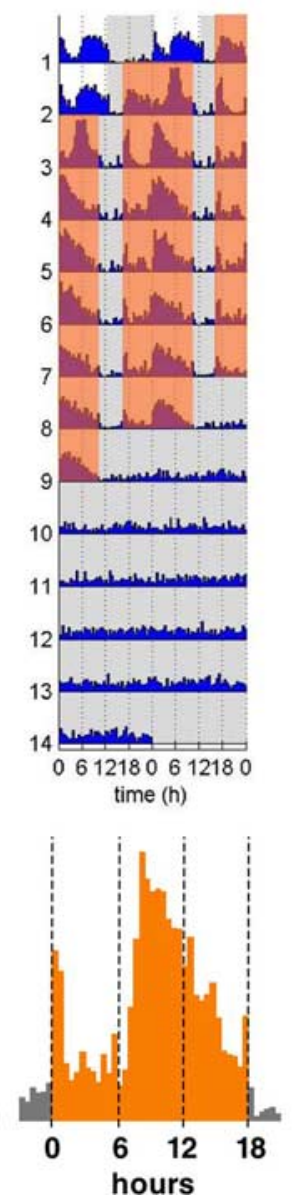

C. per $^{2}$ pdfG4-hid
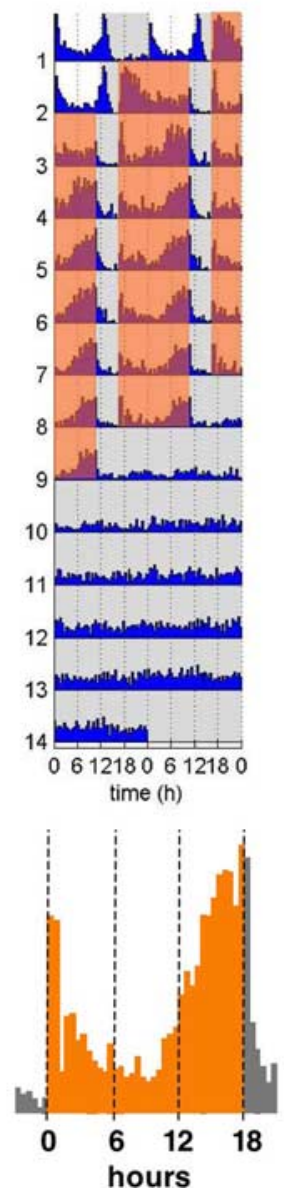

Figure 4. The evening peak is regulated by the circadian clock under TC. The M cells were genetically ablated in flies with short $(\text { per })^{S}$, long ( per $\left.{ }^{L}\right)$, or null ( per ${ }^{9}$ per alleles. After $2 \mathrm{~d}$ of $20^{\circ} \mathrm{CLD}$, the flies were exposed to a long thermophase/short cryophase TC $\left(18 \mathrm{~h}\right.$ at $29^{\circ} \mathrm{C}, 6 \mathrm{~h}$ at $20^{\circ} \mathrm{C}$, with the start of the thermophase occurring $8 \mathrm{~h}$ earlier than the lights-on transition had been during LD). The phase of the $\mathrm{M}$ cell-independent evening peak is earlier in the per ${ }^{5}$ background, later in the per background, and very abnormal in the per $^{0}$ background, demonstrating that it is under the control of the circadian clock. Average activity plots for the 3 last days in TC are shown under the actograms (orange bars, thermophase; dark gray bars, cryophase). $A, y w$; pdf-GAL4/UAS-hid; $+; n=14$; mean phase, ZT13.8 \pm 0.4. B, per'; pdf-GAL4/UAS-hid; $+; n=7$; mean phase, ZT11.0 \pm 0.4 . C, per; pdf-GAL4I UAS-hid; $+; n=12 ;$ mean phase, ZT16.5 \pm 0.2 . D, per; $;$ pdf-GAL4/UAS-hid; $+; n=10$; mean phase, ZT5.2 \pm 0.4 .ZT0 is at onset of thermophase, and mean phase refers to mean ZT of the evening (or afternoon) activity peak on the last day of TC \pm SEM. Two-tailed $t$ tests were performed comparing the phase of the evening peak in $\mathrm{M}$ cell ablated per ${ }^{S}$ and per ${ }^{l}$ flies with the phase of per $^{+}$ablated flies, and all differences were highly significant ( $p$ value $<0.001$ ).

Most LNds were missing, although we cannot entirely exclude that a subset of LNds escape ablation (the LNds are close to the DN3 groups, and one or two residual LNds could thus be mistaken for DN3 cells). Importantly, behavioral data verify that the evening peak is already completely missing during LD (Fig. $6 B$ ), which means that all E cells have been eliminated. The cry-GAL4/ UAS-hid flies showed no morning or evening peak of activity in TC, other than the brief startle response after the temperature increase (Fig. 6B). They had, however, a relatively high level of activity throughout the day.

To further confirm the role of the E cells in TC, we rescued per $^{\circ}$ flies in the M and E cells with the cry-GAL4 and UAS-per transgenes. We determined in which cells PER was expressed and found, as expected from Stoleru et al. (2004), that the LNvs showed robust rescued PER oscillations under TC (Fig. 5B). We also found that PER expression was rescued in three to four LNds
D. $p e r^{0}$ pdfG4-hid
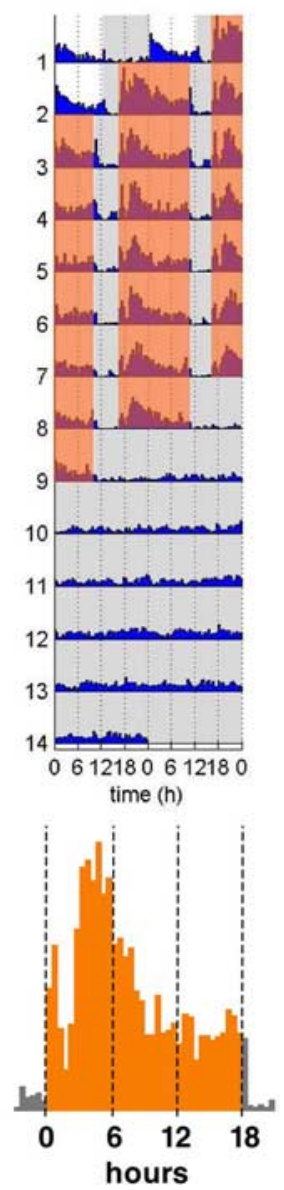

and two DN1s and that it cycled robustly. This pattern of PER expression was sufficient to fully rescue LD behavior: both the $\mathrm{M}$ and the E peak looked similar to wild type (Fig. $6 A, D$ ). In addition, like PER rescue in only the $\mathrm{M}$ cells, cry-GAL4 driven PER rescue restored rhythmicity in constant conditions after both LD and TC cycle. The circadian pacemaker thus functions normally in the $\mathrm{M}$ and $\mathrm{E}$ cells of these rescued flies, as suggested by the strong molecular oscillations observed by immunohistochemistry. However, during TC, per $^{\circ}$ flies with rescued $\mathrm{M}$ and $\mathrm{E}$ cells had a later evening peak of activity than that observed in M-cell-rescued flies, with a phase closer to that of the wild-type evening peak (Fig. 6, compare $A, C, D$ ). Thus, in TC as in $\mathrm{LD}$, the $\mathrm{E}$ cells play an important role in controlling the evening activity peak. As expected, rescuing per expression only in the $\mathrm{E}$ cells restored a similar evening peak (Fig. 6E), although for unclear reasons it appeared sharper than in flies with both the $\mathrm{M}$ and $\mathrm{E}$ cells rescued. As expected from the $\mathrm{M}$ cell ablation experiment, this peak did not persist after release in constant temperature. Therefore, the cells controlling evening activity under LD cycle play an important role in controlling evening activity under TC but cannot independently maintain rhythmicity in constant conditions.

Circadian neurons that are neither the $M$ cells nor the $E$ cells contribute to the control of circadian behavior

The previous sections demonstrate that the cells controlling circadian behavior during and after $\mathrm{LD}$ also play an important role during and after TC. However, we noted that, when PER is expressed in the $\mathrm{M}$ and E cells only (in per ${ }^{\circ}$; $c r y-G A L 4 / U A S-$ per flies), circadian behavior is not normal in phase and duration under TC (Fig. 6D). More specifically, under TC, the evening peak begins earlier and lasts longer, as if the network regulating activity is not well tuned. This abnormal behavior is not attributable to abnormal pacemaker function, because robust PER oscillations and normal LD and DD behaviors are observed in M- and E-cell-rescued per $^{\circ}$ flies (Figs. 5B, $6 D$ ). This suggests the intriguing possibility that, in wild-type flies, additional circadian neurons may contribute to regulating locomotor behavior specifically under TC.

We therefore reexamined our cry-GAL4/UAS-hid fly data and found that a small increase of activity could be detected in some of our experiments at the very end of the $12 \mathrm{~h}$ thermoperiod. However, it was difficult to distinguish this slight increase in activity from the relatively high masking activity seen when both the $\mathrm{E}$ and $\mathrm{M}$ cells are ablated (data not shown). To better visualize any residual evening activity in $\mathrm{M}$ and $\mathrm{E}$ cell ablated flies, we used the long thermoperiod/short cryoperiod assay to avoid the masking effects of cold temperature after ZT12. Interestingly, we con- 
sistently observed an evening peak of small amplitude in flies without $\mathrm{M}$ and $\mathrm{E}$ cells under $18 \mathrm{~h} / 6 \mathrm{~h}$ TC, but this peak was not present in LD with an 18-h-long photoperiod (Fig. $7 D, E$ ). Importantly, a similar peak was seen in $18 \mathrm{~h} / 6 \mathrm{~h}$ TC conditions under constant light (Fig. $7 F$ ), which demonstrates that the absence of evening activity in LD is not attributable to negative light masking. To determine whether the evening peak is of circadian nature, we monitored per mutant flies without $\mathrm{M}$ and E cells under 18 h/6 h TC conditions (Fig. 7) (supplemental Fig. S5, available at www. jneurosci.org as supplemental material). The evening peak is more apparent in the $\operatorname{per}^{S}$ and per $^{L}$ backgrounds than in wild type. Its timing is earlier in the pers background and later in $p^{L}{ }^{L}$, whereas it is absent in $\operatorname{per}^{O}$ flies. There is again no sign of evening activity in LD in any of these genotypes. Together, these data provide strong evidence that circadian neurons specifically sensitive to temperature contribute to late evening activity.

\section{The $M$ cells modulate the response of} the E cells to temperature cycles

Having demonstrated that, at physiological temperature ranges, TC cycles phase shift circadian rhythmicity much more slowly than LD cycles (supplemental Fig. S2, available at www.jneurosci.org as supplemental material), we decided to use the relative rate of entrainment to study how responsive the $\mathrm{M}$ and $\mathrm{E}$ oscillators are to temperature. We examined how flies without $\mathrm{M}$ cells synchronize to a temperature cycle. Determining the phase of behavior after TC was not possible, because these flies very rapidly become arrhythmic. We therefore measured the evening peak phase during TC in M cell ablated flies, because we have shown that this peak is controlled by the circadian clock and is not attributable to a masking phenomenon. Unexpectedly, this activity peak resynchronized very rapidly to $\mathrm{TC}$ cycles when the $\mathrm{M}$ cells were absent (Fig. 8A). After one day, the phase of the evening peak was already strongly shifted, although it varied significantly from individual to individual (Fig. $8 \mathrm{~A}$, error bars), and its amplitude was smaller than that observed after 2 or more days (data not shown). After $2 \mathrm{~d}$, virtually all flies were perfectly and fully synchronized. Therefore, intact intercellular communication from the $\mathrm{M}$ cells is necessary to modulate the response of evening oscillator clocks to temperature cycles and thus prevents circadian behavior from excessively rapidly responding to temperature inputs. This modulation requires $\mathrm{PDF}$, because flies without this neuropeptide also rapidly synchronize to temperature cycles (Fig. 8A) (supplemental Fig. S3, available at www.jneurosci.org as supplemental material).

Altering the oscillator in the M cells leads to abnormally fast entrainment to temperature cycles

The results described above strongly suggest that the $\mathrm{M}$ cells play an important role in determining the pace at which circadian behavior is synchronized by temperature. To test this hypothesis further, we exposed $y w$; $p d f$-GAL4/UAS-per flies to temperature cycles that were $8 \mathrm{~h}$ advanced relative to the LD entrainment and measured their evening peak during TC. Strikingly, synchronization to temperature cycle was considerably accelerated in these flies, which overexpress PER only in the LNvs and are otherwise wild type (Fig. $8 B$ ). This confirms that the LNvs are the cells determining the rate of synchronization to temperature, slowing it down in wild-type flies.

A likely explanation for the effect of PER overexpression on the kinetics of synchronization is a reduction in the robustness of the molecular circadian pacemaker. Excessive PER levels are known to be disruptive to circadian rhythms (Zeng et al., 1994; Kaneko et al., 2000), and a weaker oscillator is predicted to be more responsive to environmental perturbation (Pittendrigh et al., 1991). We observed that $y w$; $p d f$-GAL4/UAS-per flies have a $1 \mathrm{~h}$ longer period phenotype $(24.8 \pm 0.1)$ compared with control flies ( $y w, 23.8 \pm 0.2$ ), suggesting that the $\mathrm{M}$ cell oscillator is indeed altered. To investigate further whether oscillators with attenuated molecular oscillations are more vulnerable to temperature entrainment, we measured the rate of entrainment of $C l k^{J r k} /+$ heterozygotes, because these mutants have decreased amplitude of oscillations in per and tim transcription and PER 


\section{A. $y w$ \\ B. cry G4-hid \\ C. per pdfG4-per D. per cryG4-per E. pr \\ E. perocryG4 pdfG80-per}
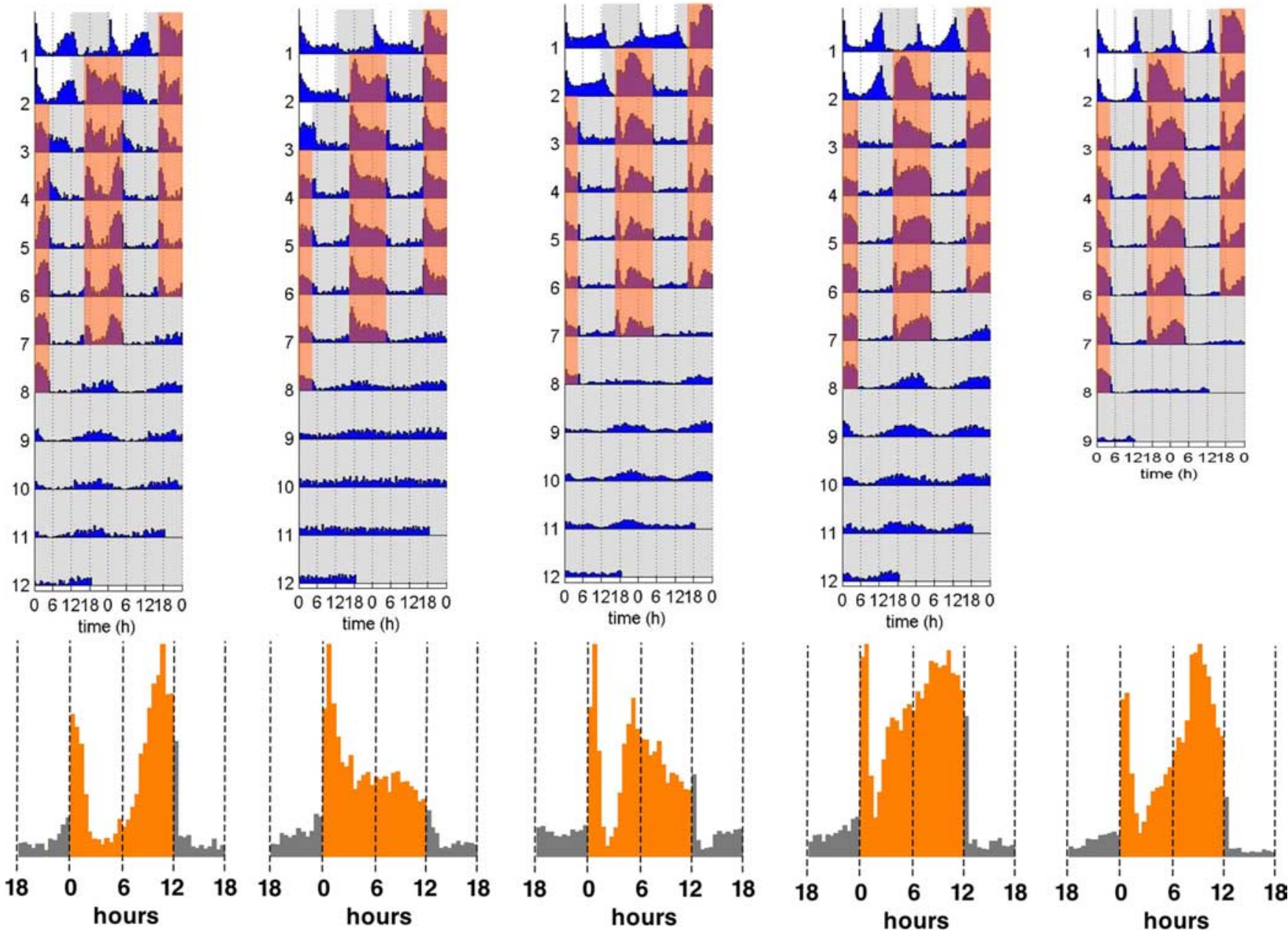

Figure 6. The PDF-negative E cells control the evening peak during temperature cycles. Flies with or without functioning $\mathrm{M}$ and $\mathrm{E}$ cells were exposed to $2 \mathrm{~d}$ of $20^{\circ} \mathrm{CLD}, 6 \mathrm{~d}$ of $29^{\circ} \mathrm{C} / 20^{\circ} \mathrm{C} \mathrm{TC}(8 \mathrm{~h}$ advanced), and then $6 \mathrm{~d}$ of CC. Average activity plots for the 3 last days in TC are shown under the actograms (orange bars, thermophase; dark gray bars, cryophase). $A$, Wild-type flies ( $y$ w; $n=12$; $67 \%$ rhythmicity after TC). B, Flies in which both the M and E cells were ablated ( $y$ w; cry-GAL4/UAS-hid; $+; n=16 ;$ no rhythmic flies after TC). C, Flies in which PER expression is limited to the M

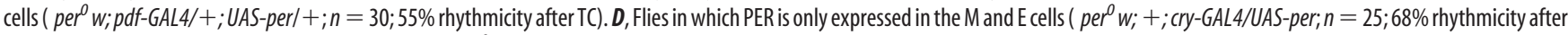
TC). $E$, Flies in which PER is only expressed in the E cells ( per $^{0} w ;$ pdf-GAL8O/+; cry-GAL4/UAS-per; $n=32$ ). The evening peak of activity cannot be detected when both the M and $\mathrm{E}$ cells are ablated $(\boldsymbol{B})$ and is abnormally early when only the $\mathrm{M}$ cells have a functional clock (C) mean phase, ZT6.4 \pm 0.3 ). When PER expression is rescued in both the $M$ and $E$ cells $(\boldsymbol{D})$, evening activity is much more prominent once stable synchronization is reached, with a later peak phase than in flies with only the $\mathrm{M}$ cells being rescued (mean phase, $278.9 \pm 0.4 ; p$ value $<10^{-5}$ ). There is also more activity during the late subjective day under constant conditions. Thus, evening activity is restored under TC, although the onset of activity is still much earlier than in wild-type flies $(\boldsymbol{A})$. Note that, during the first $2 \mathrm{~d}$ of synchronization to TC, per flies with rescued $\mathrm{M}$ and $\mathrm{E}$ cells $(\boldsymbol{D})$ show transients with a much earlier phase than after $3 \mathrm{~d}$. Experiments in which we released these flies under constant conditions after 1 or $2 \mathrm{~d}$ of entrainment revealed that, for unknown reasons, they progressively delay their rhythms rather than advancing them like wild-type flies, although the TC is advanced compared with the initial LD (data not shown). The data shown in $\boldsymbol{E}$ were obtained from a different experiment than those shown on $\boldsymbol{A}-\boldsymbol{D}$ (additional actograms for this independent experiment are shown on supplemental Fig. S4, available at www.jneurosci.org as supplemental material).

and TIM protein cycling (Allada et al., 1998). We noticed that $\mathrm{Clk}^{r k} /+$ heterozygote flies have higher activity during the cryophase than control flies (data not shown). This increase in activity partially masked the evening peak during TC entrainment. Therefore, instead of determining the phase of the evening peak during TC, we measured the phase of the free-running rhythms after 1,2,3, or $4 \mathrm{~d}$ of temperature synchronization. We found that, similarly to flies overexpressing PER in the LNvs, the $\mathrm{Clk}^{J r k} /+$ heterozygotes were much more responsive to temperature and had completely entrained to the TC cycle after $2 \mathrm{~d}$ (Fig. $8 C$ ). They even overreacted to the TC cycle, particularly on the second and third days of entrainment. Combined, our results indicate that robust molecular oscillations in the $\mathrm{M}$ cells protect Drosophila from reacting excessively to temperature cycles.

\section{Discussion}

Circadian rhythms are generated by cell-autonomous molecular pacemakers (Dunlap, 1999). In Drosophila, even circadian environmental inputs can be detected cell autonomously. Light is detected by the intracellular photoreceptor CRY in brain neurons and peripheral tissues (Emery et al., 2000). Moreover, dissected peripheral tissues can also detect temperature cycles (Glaser and Stanewsky, 2005), suggesting the existence of a cell-autonomous circadian thermosensor. It was proposed recently that the PER/ TIM dimer itself is thermosensitive (Kaushik et al., 2007).

If circadian rhythms can be synchronized and function cell autonomously, why are circadian neurons organized in a network? In Drosophila, the circadian neuronal network appears nec- 
essary to maintain stable circadian rhythms in constant conditions. Indeed, without a proper neural circuitry, individual neurons cannot keep a proper amplitude and phase for their circadian oscillations in the absence of external inputs (Peng et al., 2003; Lin et al., 2004). However, because only a minority of organisms actually experience constant conditions during their lifetime, the importance of this function under natural conditions is unclear. Recent results demonstrate the importance of the circadian neuronal network for adaptation to changes in photoperiod lengths in Drosophila (Stoleru et al., 2007) and mammals (Inagaki et al., 2007; VanderLeest et al., 2007). In Drosophila, this adaptation results from the interactions between two groups of functionally coupled circadian neurons: the $\mathrm{M}$ cells and $\mathrm{E}$ cells. Our results suggest that a robust self-sustained pacemaker is important for mitigating the resetting effects of inputs such as temperature, and further demonstrate the importance of the circadian network in the response to environmental cues. Indeed, we have identified two neuronal interactions between groups of circadian cells that are essential for proper responses to temperature cycles (Fig. 9).

The first interaction involves the aforementioned $\mathrm{M}$ and $\mathrm{E}$ cells and determines the pace at which circadian behavior is synchronized by temperature input. Drosophila behavior responds slowly to temperature cycles. Nevertheless, specific neurons can respond rapidly. The E cells are very rapidly synchronized to TC cycles if they are disconnected from the $\mathrm{M}$ cells. It is actually the $\mathrm{M}$ cells that (predominantly) set the pace of behavioral synchronization to TC, at least in DD conditions. Indeed, increasing PER levels only in the $M$ cells results in a considerably accelerated synchronization. Thus, circadian clocks can be highly sensitive to temperature input, but the pacemakers of some specific cells are more resilient. They prevent flies from overreacting to temperature changes. This is probably important in a natural environment in which Drosophila can experience erratic variations in temperature attributable to weather changes. Because the E cells can also influence the M cells (Stoleru et al., 2007), it is likely that their sensitivity to temperature cycles can be used to fine-tune the synchronization of the $M$ cells to the environment, particularly under long photoperiod.

As mentioned above, we found that specifically manipulating the circadian pacemaker of the $M$ cells accelerates synchronization to TC. We increased PER levels with the $p d f-G A L 4$ driver, which should at least double PER levels in the M cells
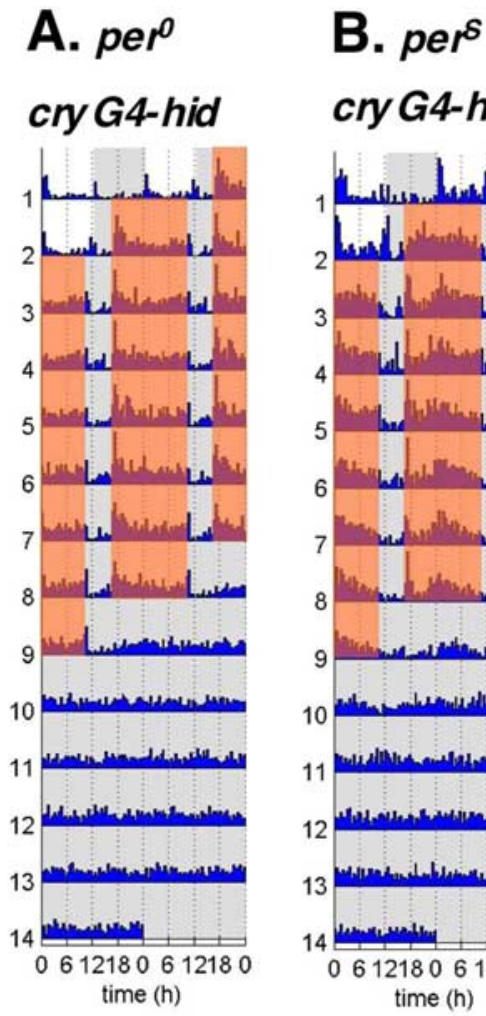

C. per ${ }^{2}$

D. per

\section{cry G4-hid}

cry G4-hid

cry G4-hid
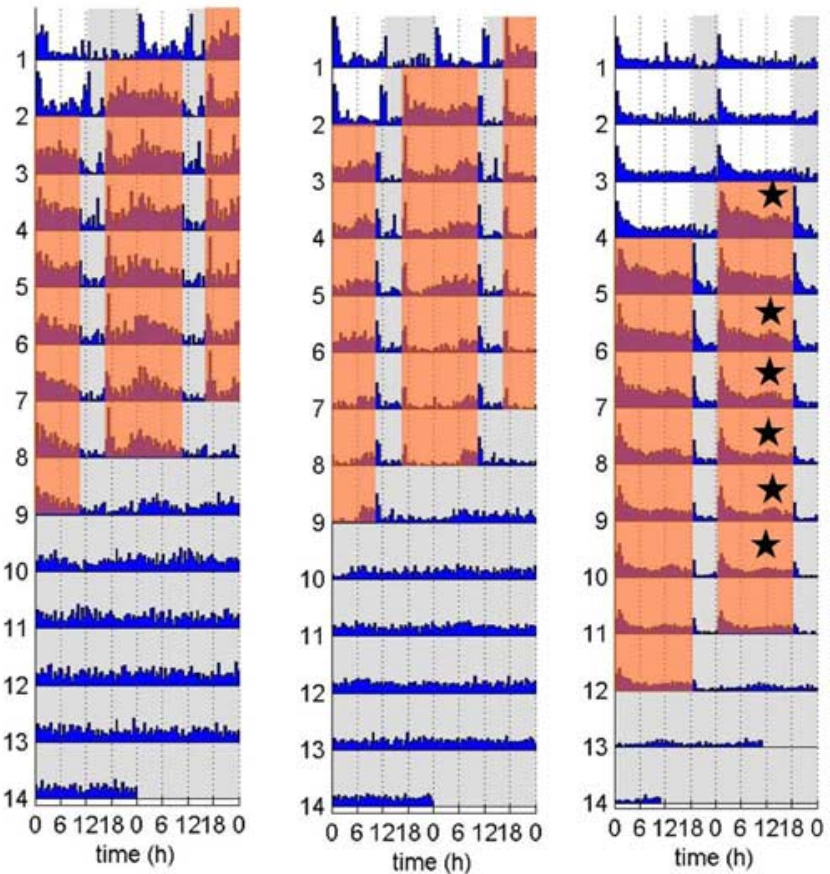

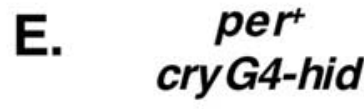

60

LD

30.

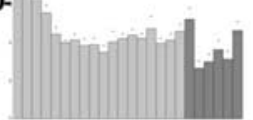

$\begin{array}{llll}0 & 6 & 12 & 18\end{array}$ hours

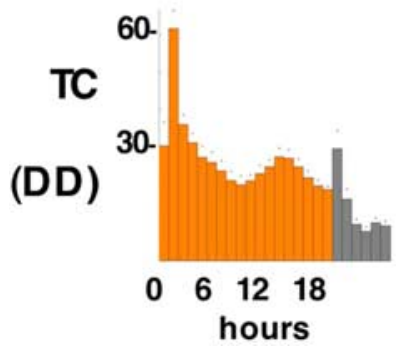

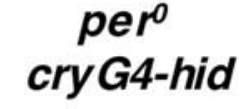

60.
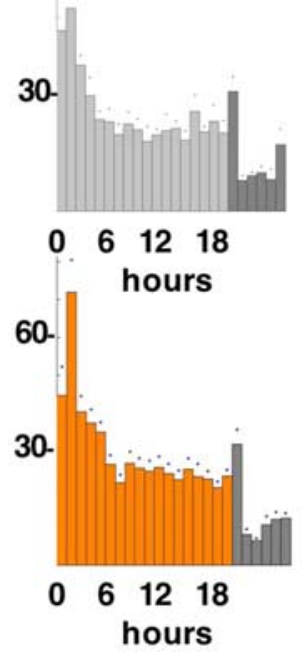

F.

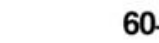

TC

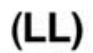

75 lux

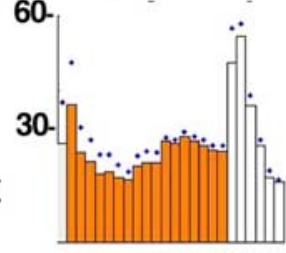

$\begin{array}{llll}0 & 6 & 12 & 18\end{array}$

hours

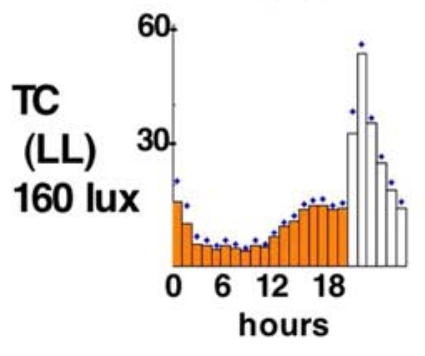

Figure 7. Neurons other than the M and $E$ cells contribute to the evening peak of activity under TC. $\boldsymbol{A}-\boldsymbol{D}$, The M and $\mathrm{E}$ cells were ablated using cry-GAL4 and UAS-hid in flies with different per alleles. per $^{0}$, per $^{5}$, and per flies were first exposed to $12 \mathrm{~h} / 12 \mathrm{~h} \mathrm{LD}$ cycles and then to TC cycles with a $16 \mathrm{~h}$ thermophase and an $8 \mathrm{~h}$ cryophase. In the case of per ${ }^{+}$, the LD and TC cycles had a $16 \mathrm{~h} \mathrm{light}$ phase and an $8 \mathrm{~h}$ dark phase $(\boldsymbol{D})$. On the per ${ }^{+}$actogram, stars indicate the evening peak when it is clearly visible. Number of flies were $8,6,10$, and 23 for per $^{0}$, per ${ }^{5}$, per ${ }^{-}$, and per $^{+}$, respectively. No rhythmicity is observed after return to constant conditions. On the fourth day of TC, mean \pm SEM phase of evening peak is ZT8.8 \pm 0.7 for per ${ }^{5}, Z T 15.5 \pm 0.7$ for per', and ZT12.0 \pm 0.4 for per ${ }^{+}$ ablated flies. Two-tailed $t$ tests were performed comparing the phase of the evening peak in ablated per ${ }^{S}$ and $p e r^{L}$ flies with the phase of per $^{+}$ablated flies, and all differences were highly significant ( $p$ value $<0.005$ ). $\boldsymbol{E}$, Average activity of per $^{+}$and per $\mathrm{r}^{0}$ flies without $M$ and E cells over $3 \mathrm{~d}$ of $16 \mathrm{~h} / 8 \mathrm{~h} \mathrm{LD}$ cycles (top graphs; gray bars, light phase; black bars, dark phase) and $6 \mathrm{~d}$ of $16 \mathrm{~h} / 8 \mathrm{~h}$ TC cycles in DD (bottom graphs; orange bars, thermophase; black bars, cryophase). For similar plots with per ${ }^{5}$ and per flies without $M$ and $E$ cells, see supplemental Figure $S 5$ (available at www.jneurosci.org as supplemental material). $\boldsymbol{F}$, Average activity of per ${ }^{+}$ flies without $M$ and $E$ cells over $6 \mathrm{~d}$ of $16 \mathrm{~h} / 8 \mathrm{~h} \mathrm{TC}$ cycles in LL at two different light intensities (orange bars, thermophase; white bars, cryophase). 


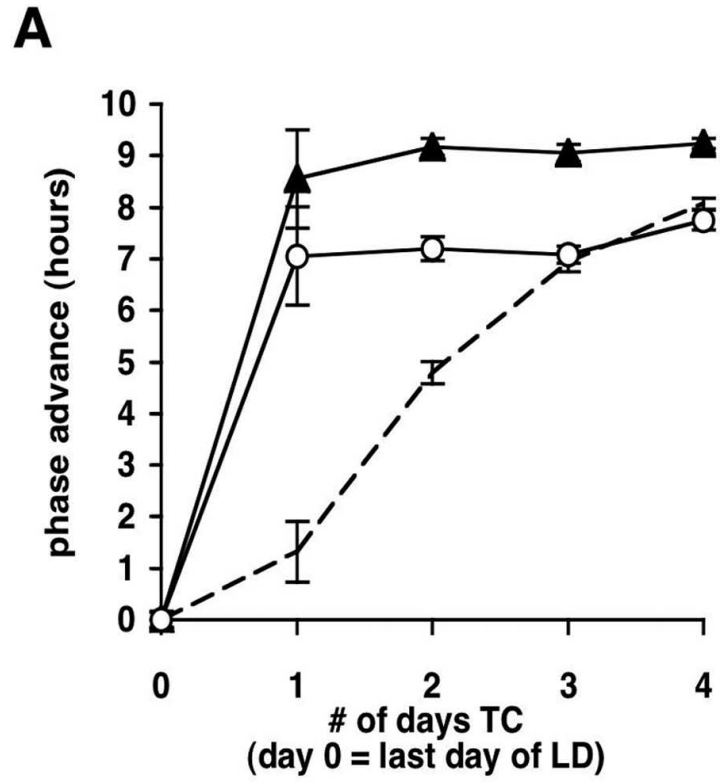

B.

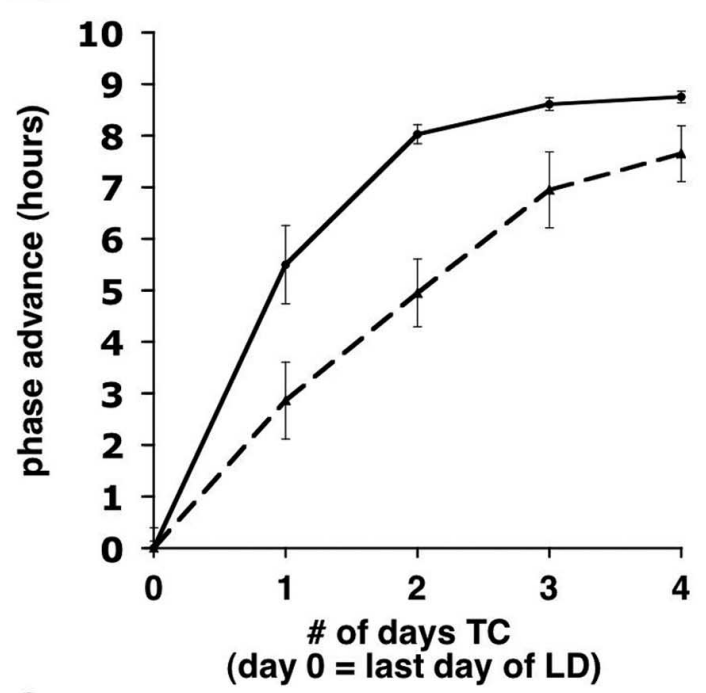

C.

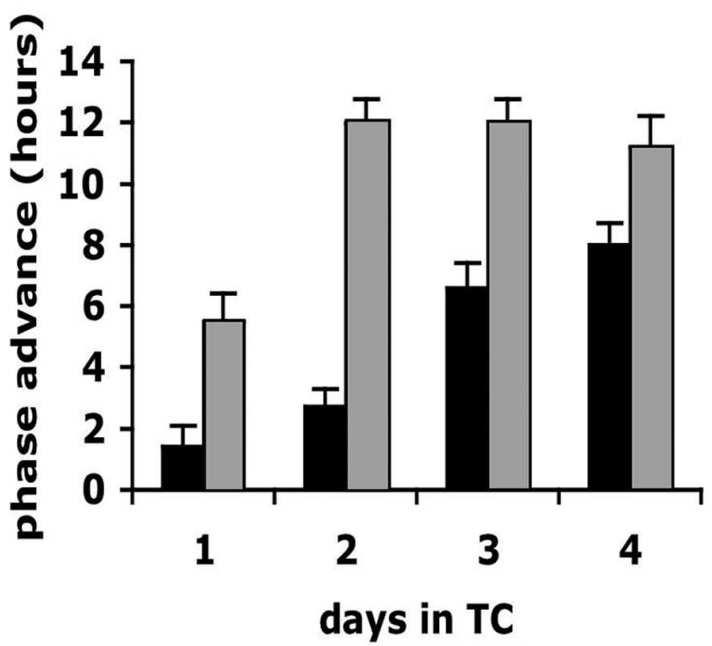

Figure 8. The E peak shows rapid synchronization in response to temperature cycles when the $\mathrm{M}$ cell oscillator is disrupted or genetically altered. $A$, Kinetics of synchronization of the cells

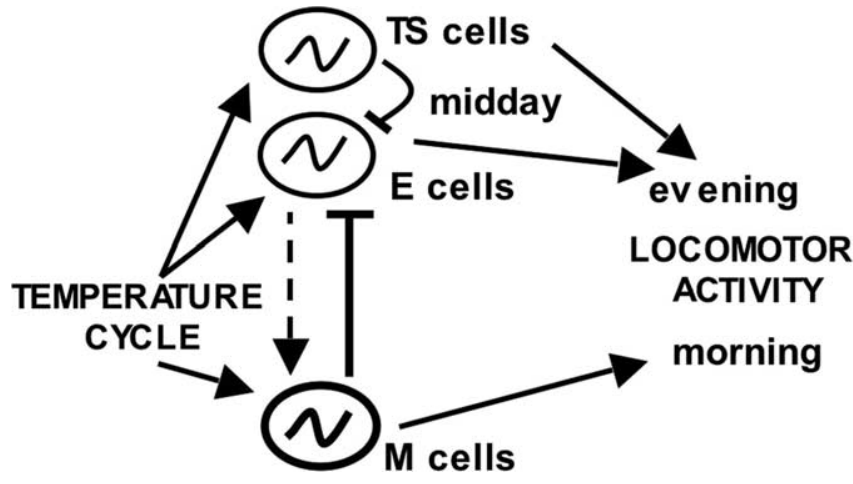

Figure 9. Model for the control of behavioral responses to temperature cycles by the circadian neuronal network. We have identified three groups of cells that contribute to behavioral responses to temperature entrainment: the $M, E$, and temperature-sensitive (TS) cells. Each group is represented by one oscillator-containing cell for simplicity. The three groups are sensitive to temperature, and they interact with each other to properly time circadian behavior in response to temperature cycles. The $M$ cells have a robust pacemaker (shown in bold) that is relatively slow at responding to temperature cycles. Through rhythmic PDF secretion, the M cells slow down the response of the highly sensitive E cells. It is however likely that the E cells can also influence the M cells (dashed arrow), particularly in the presence of light (Stoleru et al., 2007). The combination of highly sensitive $E$ cells and relatively resistant $M$ cells is probably important for the balance between behavioral adaptability to temperature changes and resistance to random variations of temperature. In addition to the $\mathrm{M}-\mathrm{E}$ cell interactions, the temperaturesensitive cells also interact with the $\mathrm{E}$ cells, inhibiting their behavioral output in the middle of the day.

(Grima et al., 2004). Our interpretation is that we have weakened the $\mathrm{M}$ cell pacemaker with this manipulation, and the $1 \mathrm{~h}$ period lengthening observed is consistent with this notion. Indeed, high PER levels increase transcriptional repression in the circadian molecular feedback loop and can in some cases completely eliminate molecular and behavioral rhythms (Zeng et al., 1994; Kaneko et al., 2000). Moreover, a weakened oscillator is predicted to respond more strongly to environmental input (Pittendrigh et al., 1991). In mammals, mutants with attenuated oscillators have stronger circadian responses to light pulses (Vitaterna et al., 2006). It should be noted that we cannot exclude that acceleration of TC synchronization is attributable at least in part to the temperature sensitivity of the GAL4/UAS system. This sensitivity could create a temperature-induced per mRNA cycling that could contribute to accelerate synchronization. However, this possibility seems unlikely. First, the kinetic of synchronization is accelerated with both advanced and delayed TC cycles (data not shown). It is unlikely that a GAL4/UAS-mediated temperature effect would affect both directions of resynchronization similarly. Second, there is little phase difference in behavior between wild-type

that regulate the evening peak to TC in wild-type flies ( $y w$; dashed line), pdf mutants ( $p d f^{01}$; solid line with open circles), and M cell ablated flies ( $y$ w; pdf-GAL4/UAS-hid; solid line with filled triangles). Flies were synchronized to $2 \mathrm{~d} L D$ and then exposed to 4 consecutive days of TC. The phase advance of the evening peak was calculated for each day in TC (in hours, relative to the phase in the last day of LD) and is plotted on the $y$-axis. $x$-Axis, Number of days under TC (day 0 corresponds to the last day of LD). Error bars indicate \pm SEM. $B$, Kinetics of TC synchronization in wild-type flies ( $y w$; dashed line) and flies with PER overexpression only in the M cells ( $y w$; pdf-GAL4; UAS-per; solid line) in $4 \mathrm{~d}$ of TC (experiment and analysis same as in $\boldsymbol{A}$ ). $C$, Kinetics of TC entrainment in wild-type flies ( $y$ w; black bars) and $C \mathrm{~K}^{\text {rlk }}$ heterozygotes ( $y$ w; + ; $\mathrm{Cl} \mathrm{K}^{\mathrm{rk}} /+$; gray bars). Because $\left(\mathrm{K}^{\text {rrk }}\right.$ heterozygotes are highly active during the cryophase under TC, phase advances were measured by comparing the phase of the evening peak after release into constant conditions ( $20^{\circ} \mathrm{CDD}$ ) in flies exposed to $1,2,3$, or $4 \mathrm{~d} \mathrm{TC}$. $y$-Axis, Phase advance (in hours) relative to no TC control flies. $x$-Axis, Total number of days in TC before release in constant conditions. Error bars indicate \pm SEM. 
and PER-overexpressing flies once stable entrainment is reached. Flies overexpressing PER only have a slightly delayed phase, which is expected because they have a $1 \mathrm{~h}$ longer period, and this would not help advancing the circadian clock under TC. Thus, any effects that the temperature sensitivity of the GAL4/UAS system could have on the phase of PER cycling is most likely superseded by the circadian regulation of PER levels and the circadian synchronization resulting from temperature cycling. In addition, we obtained independent confirmation that flies with an attenuated pacemaker are much more sensitive to temperature. Indeed, flies heterozygous for the $\mathrm{Clk}^{\mathrm{rk}}$ mutation also phase shift their clock very rapidly when exposed to a temperature cycle.

Thus, the picture emerges that a strong, self-sustained pacemaker in the $\mathrm{M}$ cells is required for proper response to temperature cycle. It keeps other oscillators controlling circadian behavior from responding excessively to temperature changes. These results fit well with those of a recent study in which flies were exposed simultaneously to temperature and light cycles, with the temperature cycle $6 \mathrm{~h}$ advanced relative to the light cycle (Miyasako et al., 2007). The PDF-positive LNvs (M cells), and the LNds and the PDF-negative LNv (E cells) followed the light cycle. Our interpretation is however different from that of Miyasako et al. (2007). These authors concluded that the $\mathrm{M}$ and $\mathrm{E}$ cells are light sensitive but not, or only weakly, temperature sensitive. First, our results show that the E cells (as defined by Stoleru et al., 2004) are actually highly sensitive to temperature cycles. Second, the $\mathrm{M}$ cells also clearly detect and respond to temperature cycles, because they are sufficient for persistent temperature synchronization. Moreover, they determine the response of circadian behavior to temperature cycles.

We also present behavioral evidence that circadian neurons distinct from the $\mathrm{M}$ and $\mathrm{E}$ cells are involved in the control of circadian behavior specifically when temperature cycles are present. An E cell- and M cell-independent peak was observed under a long thermophase TC cycle. The circadian clock controls this TC-specific peak of activity, because PER mutations displace its phase. The peak was observed under both constant darkness and constant light, indicating that its absence in an $\mathrm{LD}$ cycle is not attributable to a negative masking effect of light. Because the LPNs and the DN2s are not ablated in our experiments and are particularly sensitive to TC (Yoshii et al., 2005; Miyasako et al., 2007), they are strong candidates for playing a temperaturespecific function in the control of circadian behavior. Although it was initially proposed that the LPNs oscillate only under TC (in LL conditions) (Yoshii et al., 2005), it was recently reported that they also show molecular oscillations in LD (Shafer et al., 2006). Under our conditions, we failed to detect PER cycling in the LPNs in LD (data not shown), which might thus be of low amplitude. However, we detected robust PER cycling under TC (Fig. 5A). The LPNs therefore probably require temperature cycles to exhibit robust molecular oscillations and participate in the control of circadian rhythms.

Interestingly, non-M, non-E temperature-sensitive neurons are also necessary for properly timing the activity of the $\mathrm{E}$ cells, and this brings us to the second important circadian neuronal interaction that our study uncovered. In per $^{0}$ flies, a peak of activity is present in the middle of the day. This peak is caused by improper activity of the E cells, because it is present in per $^{0}$ flies without $\mathrm{M}$ cells but entirely disappears when both the $\mathrm{M}$ and $\mathrm{E}$ cells are ablated. When PER expression is rescued in the $\mathrm{M}$ and $\mathrm{E}$ cells of er $^{0}$ flies, circadian activity is not normal under TC, despite being perfectly rescued in LD. There are still abnormally high levels of activity in the middle of the thermoperiod. These results indicate that a group of circadian neurons are necessary for properly timing the activity of the E cells under TC. They probably inhibit the E cell output pathway, although we cannot exclude that they adjust the phase of the molecular E pacemaker. The same cells that positively participate in the evening peak under TC might be responsible for properly phasing E cell output. The LPNs are strong candidates for playing this dual role. However, it is also possible that two distinct groups of cells are responsible for activation and inhibition of locomotor behavior under TC cycles.

In conclusion, our results demonstrate that the functional coupling of different groups of circadian neurons is essential to the proper timing of behavioral activity under temperature cycles, as well as modulating the pace of synchronization so that Drosophila do not overreact to temperature changes. Our data add evidence to the emerging notion that the neural circuitry connecting circadian neurons is essential to the adaptation of behavior to the environment (Inagaki et al., 2007; Stoleru et al., 2007; VanderLeest et al., 2007).

\section{References}

Allada R, White NE, So WV, Hall JC, Rosbash M (1998) A mutant Drosophila homolog of mammalian Clock disrupts circadian rhythms and transcription of period and timeless. Cell 93:791-804.

Boothroyd CE, Wijnen H, Naef F, Saez L, Young MW (2007) Integration of light and temperature in the regulation of circadian gene expression in Drosophila. PLoS Genet 3:e54.

Brown S, A, Zumbrunn G, Fleury-Olela F, Preitner N, Schibler U (2002) Rhythms of mammalian body temperature can sustain peripheral circadian clocks. Curr Biol 17:1574-1583.

Bruce VG (1960) Environmental entrainment of circadian rhythms. In: Biological clocks, pp 29-86. Cold Spring Harbor, NY: Cold Spring Harbor Laboratory.

Dunlap JC (1999) Molecular bases for circadian clocks. Cell 96:271-290.

Edery I, Rutila JE, Rosbash M (1994) Phase shifting of the circadian clock by induction of the Drosophila period protein. Science 263:237-240.

Emery P, Stanewsky R, Helfrich-Förster C, Emery-Le M, Hall JC, Rosbash M (2000) Drosophila CRY is a deep-brain circadian photoreceptor. Neuron 26:493-504.

Glaser FT, Stanewsky R (2005) Temperature synchronization of the Drosophila circadian clock. Curr Biol 15:1352-1356.

Grima B, Chelot E, Xia R, Rouyer F (2004) Morning and evening peaks of activity rely on different clock neurons of the Drosophila brain. Nature 431:869-873.

Hardin PE (2005) The circadian timekeeping system of Drosophila. Curr Biol 15:R714-R722.

Helfrich-Förster C, Winter C, Hofbauer A, Hall JC, Stanewsky R (2001) The circadian clock of fruit flies is blind after elimination of all known photoreceptors. Neuron 30:249-261.

Inagaki N, Honma S, Ono D, Tanahashi Y, Honma K (2007) Separate oscillating cell groups in mouse suprachiasmatic nucleus couple photoperiodically to the onset and end of daily activity. Proc Natl Acad Sci USA 104:7664-7669.

Kaneko M, Hall JC (2000) Neuroanatomy of cells expressing clock genes in Drosophila: transgenic manipulation of the period and timeless genes to mark the perikarya of circadian pacemaker neurons and their projections. J Comp Neurol 422:66-94.

Kaneko M, Park JH, Cheng Y, Hardin PE, Hall JC (2000) Disruption of synaptic transmission or clock-gene-product oscillations in circadian pacemaker cells of Drosophila cause abnormal behavioral rhythms. J Neurobiol 43:207-233.

Kaushik R, Nawathean P, Busza A, Murad A, Emery P, Rosbash M (2007) PER-TIM interactions with the photoreceptor cryptochrome mediate circadian temperature responses in Drosophila. PLoS Biol 5:e146.

Konopka RJ, Benzer S (1971) Clock mutants of Drosophila melanogaster. Proc Acad Natl Sci USA 68:2112-2116.

Levine JD, Funes P, Dowse HB, Hall JC (2002) Signal analysis of behavioral and molecular cycles. BMC Neurosci 3:1.

Lin Y, Stormo GD, Taghert PH (2004) The neuropeptide pigment- 
dispersing factor coordinates pacemaker interactions in the Drosophila circadian system. J Neurosci 24:7951-7957.

Liu Y, Merrow MW, Loros JJ, Dunlap JC (1998) How temperature changes reset a circadian oscillator. Science 281:825-829.

Majercak J, Sidote D, Hardin PE, Edery I (1999) How a circadian clock adapts to seasonal decreases in temperature and day length. Neuron 24:219-230.

Miyasako Y, Umezaki Y, Tomioka K (2007) Separate sets of cerebral clock neurons are responsible for light and temperature entrainment of Drosophila circadian locomotor rhythms. J Biol Rhythms 22:115-126.

Murad A, Emery-Le M, Emery P (2007) A subset of dorsal neurons modulates circadian behavior and light responses in Drosophila. Neuron 53:689-701.

Peng Y, Stoleru D, Levine JD, Hall JC, Rosbash M (2003) Drosophila freerunning rhythms require intercellular communication. PLOS Biol 1:E13.

Pittendrigh CS (1954) On temperature independence in the clock-system controlling emergence time in Drosophila. Proc Natl Acad Sci USA 40:1018-1029.

Pittendrigh CS (1967) Circadian systems. I. The driving oscillation and its assay in Drosophila pseudoobscura. Proc Natl Acad Sci USA 58:1762-1767.

Pittendrigh CS, Kyner WT, Takamura T (1991) The amplitude of circadian oscillations: temperature-dependence, latitudinal clines, and the photoperiodic time measurement. J Biol Rhythms 6:299-313.

Renn SCP, Park JH, Rosbash M, Hall JC, Taghert PH (1999) A pdf neuropeptide gene mutation and ablation of PDF neurons each cause severe abnormalities of behavioral circadian rhythms in Drosophila. Cell 99:791-802.

Rieger D, Shafer OT, Tomioka K, Helfrich-Förster C (2006) Functional analysis of circadian pacemaker neurons in Drosophila melanogaster. J Neurosci 26:2531-2543.

Shafer OT, Helfrich-Förster C, Renn SC, Taghert PH (2006) Reevaluation of Drosophila melanogaster's neuronal circadian pacemakers reveals new neuronal classes. J Comp Neurol 498:180-193.

Stanewsky R, Kaneko M, Emery P, Beretta M, Wager-Smith K, Kay SA, Rosbash M, Hall JC (1998) The cryb mutation identifies cryptochrome as a circadian photoreceptor in Drosophila. Cell 95:681-692.
Stoleru D, Peng Y, Agusto J, Rosbash M (2004) Coupled oscillators control morning and evening locomotor behaviour of Drosophila. Nature 431:862-868.

Stoleru D, Peng Y, Nawathean P, Rosbash M (2005) A resetting signal between Drosophila pacemakers synchronizes morning and evening activity. Nature 438:238-242.

Stoleru D, Nawathean P, Fernandez Mde L, Menet JS, Ceriani MF, Rosbash M (2007) The Drosophila circadian network is a seasonal timer. Cell 129:207-219.

VanderLeest HT, Houben T, Michel S, Deboer T, Albus H, Vansteensel MJ, Block GD, Meijer JH (2007) Seasonal encoding by the circadian pacemaker of the SCN. Curr Biol 17:468-473.

Vitaterna MH, Ko CH, Chang AM, Buhr ED, Fruechte EM, Schook A, Antoch MP, Turek FW, Takahashi JS (2006) The mouse Clock mutation reduces circadian pacemaker amplitude and enhances efficacy of resetting stimuli and phase-response curve amplitude. Proc Natl Acad Sci USA 103:9327-9332.

Wheeler DA, Hamblen-Coyle MJ, Dushay MS, Hall JC (1993) Behav in light-dark cycles of Drosophila mutants that are arrhythmic, blind, or both. J Biol Rhythms 8:67-94.

Yoshii T, Sakamoto M, Tomioka K (2002) A temperature-dependent timing mechanism is involved in the circadian system that drives locomotor rhythms in the fruit fly Drosophila melanogaster. Zoolog Sci 19:841-850.

Yoshii T, Funada Y, Ibuki-Ishibashi T, Matsumoto A, Tanimura T, Tomioka K (2004) Drosophila cryb mutation reveals two circadian clocks that drive locomotor rhythm and have different responsiveness to light. J Insect Physiol 50:479-488.

Yoshii T, Heshiki Y, Ibuki-Ishibashi T, Matsumoto A, Tanimura T, Tomioka K (2005) Temperature cycles drive Drosophila circadian oscillation in constant light that otherwise induces behavioural arrhythmicity. Eur J Neurosci 22:1176-1184.

Zeng H, Hardin PE, Rosbash M (1994) Constitutive overexpression of the Drosophila period protein inhibits period mRNA cycling. EMBO J 13:3590-3598.

Zimmerman WF, Pittendrigh CS, Pavlidis T (1968) Temperature compensation of the circadian oscillation in Drosophila pseudoobscura and its entrainment by temperature cycles. J Insect Physiol 14:669-684. 